\title{
An Energy Efficient Routing Protocol Based on Layers and Unequal Clusters in Underwater Wireless Sensor Networks
}

\author{
Fang Zhu $\mathbb{D}^{1}$ and Junfang Wei ${ }^{2,3}$ \\ ${ }^{1}$ School of computer and communication engineering, Northeastern University at Qinhuangdao, Northeastern University, \\ Qinhuangdao 066004, China \\ ${ }^{2}$ School of Resources and Materials, Northeastern University at Qinhuangdao, Northeastern University, Qinhuangdao 066004, China \\ ${ }^{3}$ Key Laboratory of Dielectric and Electrolyte Functional Material Hebei Province, School of Resource and Materials, \\ Northeastern University at Qinhuangdao, Qinhuangdao 066004, China
}

Correspondence should be addressed to Fang Zhu; sky050607@sina.com

Received 3 August 2018; Accepted 13 November 2018; Published 16 December 2018

Academic Editor: Abdellah Touhafi

Copyright (C) 2018 Fang Zhu and Junfang Wei. This is an open access article distributed under the Creative Commons Attribution License, which permits unrestricted use, distribution, and reproduction in any medium, provided the original work is properly cited.

\begin{abstract}
Underwater Wireless Sensor Networks (UWSNs) have drawn tremendous attentions from all fields because of their wide application. Underwater wireless sensor networks are similar to terrestrial Wireless Sensor Networks (WSNs), however, due to different working environment and communication medium, UWSNs have many unique characteristics such as high bit error rate, long end-to-end delay and low bandwidth. These characteristics of UWSNs lead to many problems such as retransmission, high energy consumption and low reliability. To solve these problems, many routing protocols for UWSNs are proposed. In this paper, a localization-free routing protocol, named energy efficient routing protocol based on layers and unequal clusters (EERBLC) is proposed. EERBLC protocol consists of three phases: layer and unequal cluster formation, transmission routing, maintenance and update of clusters. In the first phase, the monitoring area under the water is divided into layers, the nodes in the same layer are clustered. For balancing energy of the whole network and avoiding the "hotspot" problem, a novel unequal clustering method based on layers for UWSNs is proposed, in which a new calculation method of unequal cluster size is presented. Meanwhile, a new cluster head selection mechanism based on energy balance and degree is given. In the transmission phase, EERBLC protocol proposes a novel next forwarder selection method based on the forwarding ratio and the residual energy. In the third phase, Intra and inter cluster updating method is presented. The simulation results show that the EERBLC can effectively balance the energy consumption, prolong the network lifetime, and increase the amount of data transmission compared with DBR and EEDBR protocols.
\end{abstract}

\section{Introduction}

Nowadays, Underwater Wireless Sensor Networks (UWSNs) are attracting more attentions from academia and industry because of their broad application fields such as environmental monitoring, disasters prevention, auxiliary navigation, resource exploration and so on [1-7]. UWSN is composed of the base station, sink nodes and ordinary sensor nodes. The sensor nodes are randomly deployed from surface to bottom of water. They collect and transmit the information to the sink nodes. Sink nodes receive the information form sensor nodes, and then send the information to the base station. Sink nodes are usually deployed on the surface of water. The base station processes the data and supports the final decision through data analysis. Figure 1 shows the architecture of UWSNs. UWSNs are similar to terrestrial Wireless Sensor Networks (WSNs). However, considering the physical layer technology and propagation medium, the challenges are very different from terrestrial WSNs. In the terrestrial WSNs, data are transmitted through radio signals. But it is not suitable for underwater environment, due to rapid decay and high energy consumption in the water. Usually in the water, the acoustic signal is adopted as communication medium. The speed of acoustic signal is significantly 


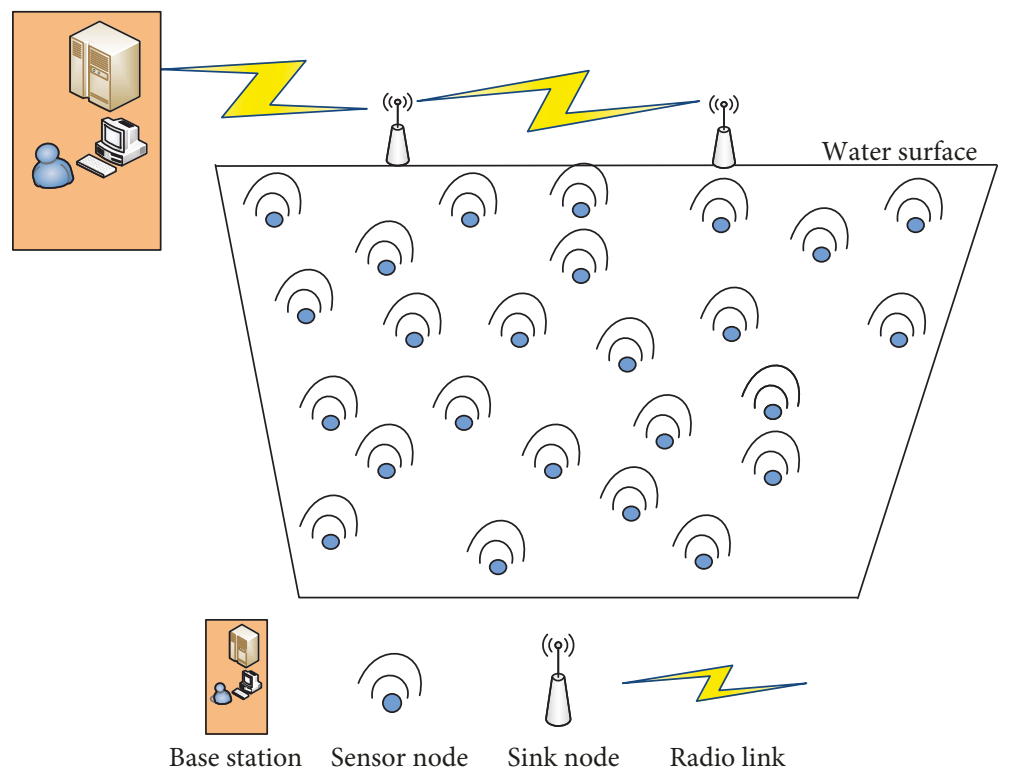

FIgURE 1: Architecture of UWSN.

slower than radio signal, which causes the high propagation delay. Moreover, multipath fading channel causes high bit error ratio and the low bandwidth. These characteristics lead to many problems such as retransmission, high energy consumption and low reliability. Therefore, design of routing protocols suitable for UWSNs becomes a challenge task.

Many routing protocols have been proposed for UWSNs in last few years [8-29]. These protocols can be classified into two categories: localization-based and localization-free routing protocols. In the section 2 , we introduce two types of protocols in detail. In the localization-based protocols, each node requires to know its location. However, location-known is a hard issue for UWSNs because GPS device is not suitable for working in the water and the position of node is changing at any time with water current. Localization-free routing protocols are more suitable for UWSNs than localization-based routing protocols, because the nodes do not need to know their location in localization-free routing protocols.

In this paper, we propose a localization-free routing protocol, named energy efficient routing protocol based on layers and unequal clusters (EERBLC). This routing protocol aims to solve the problems of high energy consumption, long propagation delay and high error rate. In this protocol, the monitoring area is layered according to the distance to the surface, and then nodes are clustered in each layer. In this process, unequal clusters are formed to balance energy. The size of cluster closer to the surface is smaller than far away from the surface. EERBLC protocol presents layer algorithm, cluster head election algorithm and cluster size calculation algorithm. Cluster member nodes collect and send the data to cluster head node in each cluster. Cluster head nodes perform data aggregation and forward the data to sink nodes by multi-hop path. Furthermore, a novel approach of selecting the next-hop based on link quality and residual energy is proposed during the data forwarding. Finally, the method of maintenance and update of cluster is presented in EERBLC protocol.
The rest of this paper is organized as follows. Some well-known routing protocols proposed for UWSNs are summarized in Section 2. Section 3 describes the proposed routing protocol EERBLC in detail. Section 4 presents the performance evaluation of EERBLC. Finally, Section 5 illustrates the conclusion of this study.

\section{Related Works}

UWSNs have been under research over last ten years. Many routing protocols for UWSNs have been proposed by some researchers. As mentioned above, these protocols can be classified into two categories: localization-based and localization-free.

2.1. Localization-Based Routing Protocols. A vector-based forwarding (VBF) routing protocol is proposed in literature [14]. In this protocol, it supposes that each sensor node knows its own location. Sensor nodes forward data packets with the location information of source, destination and a range field. Only those nodes in a pipe with a given radius can be selected as forwarders. The pipe is halved by a vector from the source node to the sink node. VBF reduces the number of forwarding nodes, so the overhead of whole network is reduced. However, it is sensitive to the radius of pipe. Based on the VBF protocol, many improved protocols are proposed [15-17]. Some of these protocols [15, 16] improve the delivery ratio, and some [17] improve the energy consumption. Focused Bream Routing (FBR) is presented to reduce unnecessary flooding in literature [18]. During the selection of forwarder, FBR uses different transmission power levels. In the FBR protocol, the mechanism of RTS/ CTS leads to long end-to-end delay and excessive energy consumption. A novel multipath grid-based geographical routing protocol (EMGGR) for UWSNs is proposed in literature [19]. EMGGR routing protocol assumes that the geographic area of the network is partitioned into $3 \mathrm{D}$ logical 
grids and sensor nodes are deployed in some cells. It selects gateways based on their locations and remaining energy level. Some protocol similar to EMGGR are proposed such as NGF [11], GGFGD and GFGD [20]. Literature [21] proposes the depth-controlled routing (DCR). In DCR, each sensor node with network topology controller can adjust the its depth to organize the network topology when the greedy geographic routing fails. DCR is the first geographic routing protocol for UWSNs that considers the sensor node vertical movement ability to move it for topology control purpose.

2.2. Localization-Free Routing Protocols. In the localizationbased routing protocols, full-dimensional location information is needed. However, in the underwater environment, the location information is hard to acquire. So, many localization-free routing protocols were proposed.

A classical localization-free routing protocol called depth-based routing (DBR) is presented in literature [22]. This protocol assumes that each node equipped with a depth sensor. DBR selects next forwarder based on depth information. DBR is a practical routing protocol. However, it has some serious problems. First, if the nodes have same depth, they will forward the data packet at same time. Even if the depths are not exactly the same, because of the long propagation delay in UWSNs, the same packet can be forwarded by different nodes. Particularly, in density areas, large number of redundant data packets will be generated which leads to high energy consumption. Second, in the case of sparse areas, it is possible that no sensor node can be selected as forwarder due to its greedy mode. Third, because each node needs to keep data packet for a certain time after receiving data packet, it causes long end-to-end delay. These problems affect the performance of DBR. To improve DBR's energy consumption problem, Energy-efficient depth-based routing protocol (EEDBR) for UWSNs is proposed in literature [23]. EEDBR improvs the performance in terms of the network lifetime, energy consumption and end-to-end delay. Hop-by-hop dynamic addressing-based routing protocol (H2-DAB) is proposed in literature [24]. In H2-DAB, each node needs to be assigned a HopID. The HopID of node represents hop count from current node to sink nodes. The nodes with smallest HopID are selected as forwarders by source node. That causes the problem of void region. Moreover, the inquiry request and reply mechanism results in long endto-end delay and extra energy consumption. To improve the delivery ratio, some localization-free protocols are proposed such as CARP and E-CARP $[12,25]$. In literature [26], a cluster-based routing protocol (CBKU) for UWSNs is proposed. CBKU uses the improved $\mathrm{K}$-means algorithm for clustering to avoid energy unbalanced. To prolong the lifetime of UWSNs, some location-free protocols are proposed based on cluster [27-29].

\section{Design of Proposed Protocol (EERBLC)}

3.1. Motivation of EERBLC. Reducing energy consumption is the main objective of a routing protocol for UWSNs. In underwater environment, acoustic signal is adopted as communication medium, which leads to more energy consumption. The energy of sensor nodes is limited and hard to be supplied. Hence, energy efficient and energy balance are primary design objectives in a routing protocol. Cluster-based routing protocols have been proposed in the terrestrial wireless sensor networks [30-33]. After clustering, member sensor nodes collect and send data to the cluster head, cluster head sends the data to the sink node after data fusion. It has been proved that cluster-based protocols are very effective on saving energy. In UWSNs, transmission requires more energy than receiving. Therefore, reducing the number of transmissions is useful in reducing the energy consumption. Cluster head aggregates and fuses data can effectively reduce the number of transmission. Because long distance communication leads to more energy consumption, multi-hop path routing method is adopted to save the energy in our protocol. Cluster heads forward the data to sink nodes by other cluster heads. Therefore, our work aims to design a routing protocol more suitable for UWSNs based on cluster.

Some protocols for UWSNs have introduced clustering techniques [34-38], but none of them takes into account the "hotspot" problem. "Hotspot" problem is that cluster heads especially the nodes near the surface forward data more frequently than others, which results in premature death of these nodes. The "hotspot" problem affects whole network lifetime. Therefore, EERBLC protocol aims to improve the clustering technique to solve the hotspot problem. The idea is that the nodes near the surface are not clustered, and each node of these can be selected as forwarder; At the same time, unequal cluster technology is applied to avoid the "hotspot" problem. The layer is closer to the surface, the number of cluster is lager and size of cluster is smaller. Furthermore, during the selection of the forwarder, energy balance should be considered.

Due to the harsh environment, the bit error rate is very high and delivery ratio is very low in UWSNs. High quality links can improve bit error rate, delivery ratio, and energy consumption. Therefore, the selection of reliable routing path with good link quality is very important issue. During the selection of next forwarder, the link quality and residual energy are both taken into account in EERBLC protocol.

3.2. Network Structure and Assumption. The network structure is shown in Figure 1. EERBLC adopts multi-sinks mode to increase the reliability of the network and the data delivery ratio. This mode also can reduce the energy consumption of the nodes around the sinks. Sink nodes are distributed evenly on the water surface. Each sink node is equipped with a radio modem and acoustic modem. Radio modem is used to communicate between sink nodes and the data center. Acoustic modem is used to communicate between sensor nodes under the water.

To make the clustering more effective, Underwater sensor nodes are deployed in the form of layer from bottom to surface in a $3 \mathrm{D}$ space. The sensor nodes are deployed at different layers by a bouncy control mechanism. The numbers of layers depended on the depth of the water and the communication range between the layers. If the average depth of the ocean is about $2.5-3 \mathrm{~km}$ and the communication rang is 
$500 \mathrm{~m}$, about 5-6 layers are needed. Each sensor node is equipped with an acoustic modem for communicating with each other in the underwater. Underwater sensor nodes drift with water current in the horizontal direction, the vertical movement can be negligible.

All underwater sensor nodes can sensor and collect data. In each layer, the nodes are clustered except in the highest layer where the nodes can directly communicate with sink nodes. In each cluster, cluster members send the data to the cluster head. Cluster head aggerates and forwards the data to other cluster head. The data are sent to the sink by multi-hop path. Considering the energy balance, clustering is not needed in the highest layer, where all nodes can be selected as forwarder.

In this paper, we assume the energy of sink nodes is unlimited because the battery of sink nodes can be replaced. Meanwhile, we assume the underwater sensor nodes have equal initial energy and same communication range. And the energy of underwater sensor nodes is one-time. Each underwater sensor node equipped with the depth sensor. Once any one of sink nodes receives the data packets, delivery is considered to be successful.

3.3. Energy Consuming Model. Due to the characteristics of underwater acoustic channel, the energy consuming model of UWSNs is quite different from the energy consuming model of terrestrial WSNs. The formula (1) shows the calculation method.

$$
E(d, f)=P_{0} d^{k} a^{d}
$$

Where $P_{0}$ is the power threshold that the data can be received by the nodes, $\mathrm{d}$ is the transmitting distance, $\mathrm{k}$ is the coefficient of energy expansion, $E(\mathrm{~d}, f)$ is the lowest energy consumption to send the data to the destination. $a$ is defined as formula (2).

$$
a=10^{a(f) / 10}
$$

Where $a$ is concerned with frequency, which is defined as formula (3).

$$
a(f)=0.11 \frac{f^{2}}{1+f^{2}}+44 \frac{f^{2}}{4100+f^{2}}+2.75 \times 10^{-4} f^{2}+0.003
$$

Where $f$ is the frequency of the carrier acoustic signal in $\mathrm{Hz}$ and $a(f)$ is in $\mathrm{dB} / \mathrm{m}$.

$E_{D A}(l)$ means the energy consumed by fusing 1 bits of data, which is shown as formula (4).

$$
E_{D A}(l)=E_{E A} * l
$$

Where $E_{E A}$ is the energy consumed by fusing one bit of data, generally it can be taken as $5 \mathrm{~nJ} / \mathrm{bit}$.

3.4. EERBLC Protocol. As mentioned above, in the EERBLC protocol, the underwater sensor nodes are deployed in form of layer. For example, if the depth of monitoring area is $1000 \mathrm{~m}$ and the communication rang of nodes is $250 \mathrm{~m}$, the whole network needs 4 layers of nodes. The distance is less than or equal to $250 \mathrm{~m}$ between two layers. In each layer, the sensor nodes are randomly deployed. The nodes compete to be cluster head at the same layer.

There are three phases in the EERBLC protocol, including cluster formation, transmission routing and maintenance and update of clusters.

3.4.1. Cluster Formation. The multi-hop transmission causes an unbalanced energy consumption. Cluster heads closer to the sink nodes take on more forwarding tasks than other cluster heads. To solve this problem, the unequal cluster technology is adopted in EERBLC protocol. Clusters will have equal cluster sizes at the same layer. The size of cluster with higher depth is larger. In the first layer, cluster is not formed and each node can forward the data to sink nodes directly. The structure of the network in EERBLC protocol is shown in Figure 2. In order to balance energy consumption between nodes in each cluster, the cluster heads selection mechanism is based on three parameters: residual energy, the degree and the layer number.

After sensor nodes are deployed, each node calculates its own layer number. The formula (5) shows the calculation method of layer number.

$$
L n=\left(\frac{n_{i \_d e p t h}}{R}\right)+1
$$

Where Ln is layer number of node $n_{i}, n_{i-\text { depth }}$ is the depth of $n_{i}, \mathrm{R}$ is the maximum communication rang of node.

There are three steps in the cluster setup phase. The first step of this phase is information collection phase, whose duration is set as T1. At the beginning of this phase, each node broadcasts a N_HELLO message which includes node id, layer number, residual energy. Once receiving the N_HELLO message, each node records the message of neighbors at the same layer. The message from nodes at other layers is dropped. The format of the N_HELLO packet is shown in Table 1.

The second step is the cluster head competition phase, which starts when T1 expires. In this phase, the sensor node whose waiting time Tc expires becomes cluster head, and broadcasts a competition message N_COM. The competition message N_COM maintains node id, layer number, residual energy and degree. Degree is the number of neighbors. The format of the N_COM packet is shown in Table 2.

If the node $S_{i}$ received $N_{-}$COM message from other nodes in the same layer before Tc expires, node $S_{i}$ drops competition and becomes an ordinary node. Tc is different for each node, which is calculated as following formula (6).

$$
T c=\left(1-\frac{\text { residual energy }}{\text { initial energy }}\right) * T 2+P
$$

Where T2 is the duration of the competition, $P$ is the random value in $[0.5,1]$, which is used to avoid the 


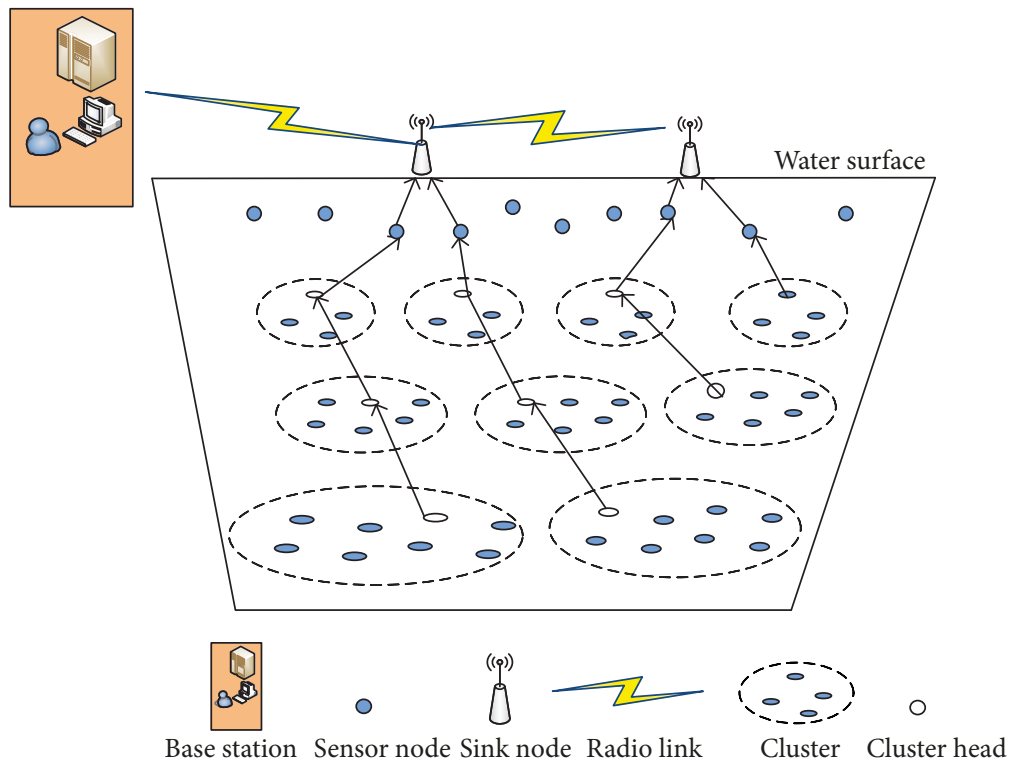

Figure 2: Structure of the network in EERBLC.

TABLE 1: Format of N_HELLO packet.

\begin{tabular}{llll}
\hline Type of packet & Node id & Layer number & Residual energy \\
\hline
\end{tabular}

TABLE 2: Format of N_COM packet.

Type of packet Node id Layer number Residual energy Degree

communication conflict when residual energy of nodes is the same. It can be seen from formula (6) that the nodes with higher residual energy have shorter waiting time and have a greater chance to be cluster head. Once the waiting time Tc of a node expires, it is selected as candidate cluster head and broadcasts N_COM message within competition communication rang.

In order to balance energy of whole network, unequal clusters are formed in different layers. The nodes closer to the surface have heavier forwarding load. Hence, the cluster size is smaller in the layer which is closer to surface. In order to generate unequal clusters, these nodes need to calculate their own competition radius Rc. The formula (7) shows the computing method of Rc.

$$
R c=\beta \frac{\text { energy }_{c}}{\text { energy }_{\text {init }}} *\left(\alpha \frac{\operatorname{Ln}}{\operatorname{Ltn}} * R\right)
$$

Where $L n$ is the layer number of node, $L t n$ is total layer number of whole network, energy $y_{c}$ is current residual energy, $\alpha$ and $\beta$ is weighted value. It is shown from formula (7) that nodes with bigger layer number have lager competition radius. And the nodes with more energy have lager competition radius at the same layer.

If a node $S_{j}$ receives a N_COM message from node $S_{i}$ before its waiting time Tc expires, it adds the $S_{i}$ to its candidate cluster head list and becomes a non-cluster head node. If the node receives many N_COM messages from several
TABLE 3: Format of N_JOIN packet.

Type of packet Head node id Member id Residual energy

cluster heads, it requires to select a cluster head from its candidate cluster head list. To optimize the selection method of cluster head, we design a multi-objective optimization technique to calculate the cost value for each candidate. The cost value is calculated as formula (8).

$$
\cos t(i)=\gamma \frac{\text { energy }_{\text {res } \_i}}{\text { energy }_{\text {init }}}+\theta \frac{{\operatorname{deg} r e e_{-} i}_{N}}{N}, \gamma+\theta=1
$$

Where energy ${ }_{\text {res_i }}$ is the residual energy of candidate $\mathrm{S}_{\mathrm{i}}$, $\operatorname{deg} \mathrm{ree}_{-}{ }_{i}$ is the number of neighbors of candidate $\mathrm{S}_{\mathrm{i}}, \mathrm{N}$ is the total number of all nodes. $\gamma$ and $\theta$ are the parameters which are used to adjust the weight of energy and degree. The node with maximum cost value will be selected as cluster head by node $S_{j}$. If there are several cluster head nodes with the maximum value, the first node with maximum value in the list is selected as its cluster head. After selection of cluster head, node $S_{j}$ replies a N_JOIN message to the selected head node. N_JOIN maintains id of head node, id of $S_{j}$, residual energy. The head node adds $S_{j}$ to its member list. The format of the N_JOIN packet is shown in Table 3.

The procedure of cluster formation is shown as algorithm 1 .

3.4.2. Transmission Routing Algorithm. After cluster formation phase, each cluster head assigns a TDMA schedule to each member of its cluster. Each member node transmits its data at its allocated time slot. The cluster head aggregates and forwards the data to the sink nodes. If the node resides at the highest layer which number is 1, the data are transmitted to the sink nodes directly. In other case, the data are forwarded by cluster heads through multi-hop path. 


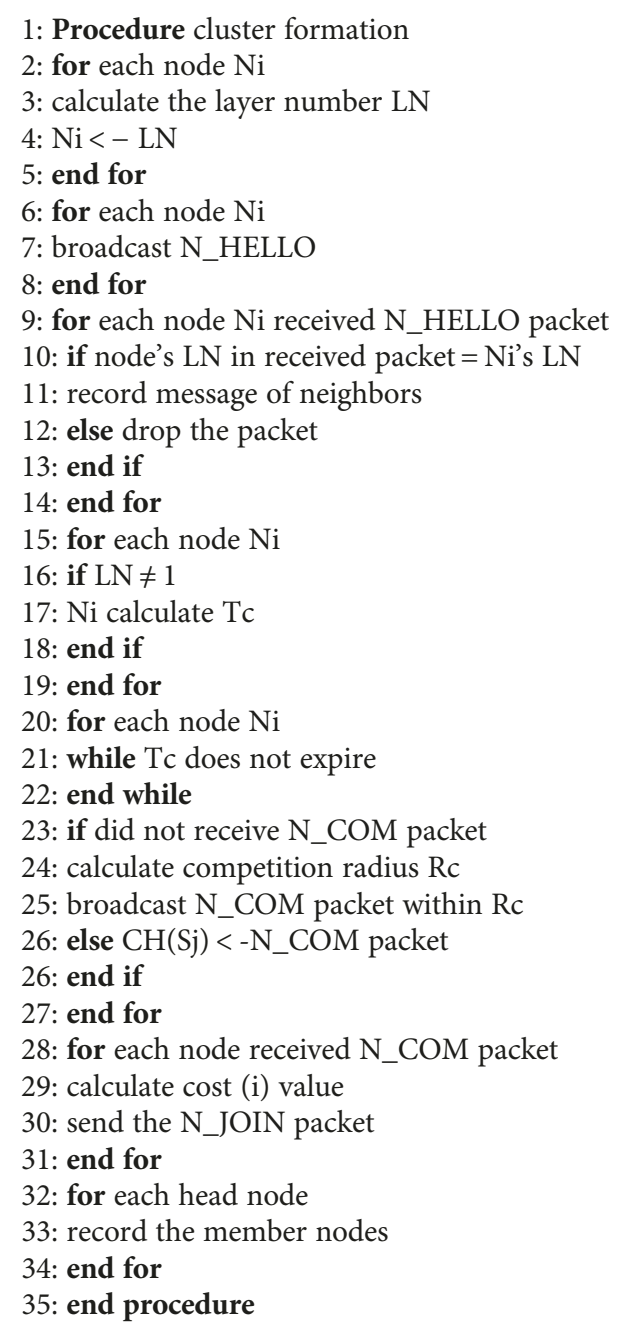

Algorithm 1: Cluster formation algorithm.

Each cluster head records the information of head id, residual energy and forwarding ratio when it hears data packets from other heads at its upper layer which layer number is one less than its layer number. Cluster head only selects the nodes at upper layer as next forwarder. Considering the energy efficient and delivery ratio, the selection of next hop is based on residual energy and forwarding ratio. The cost value of selection is computed as following formula (9).

$$
H_{-} \cos t(i)=\mu \frac{\text { energy }_{\text {res_Hi }}}{\text { energy }_{\text {init }}}+\varepsilon f r, \mu+\varepsilon=1
$$

Where energy $y_{\text {res_Hi }}$ is the residual energy of head node $\mathrm{S}_{\mathrm{i}}$, fr is the forwarding ratio of $\mathrm{S}_{\mathrm{i}}, \mu$ and $\varepsilon$ are the weight coefficient.

Cluster head calculates cost value of each cluster head according to the information recorded. For improving deliver ratio, two head nodes with the biggest value are selected as next forwarder. Cluster heads forward data packet including data number, source id, head id, next hop id1, next hop id2, data, residual energy and forwarding ratio. The format of data packet is shown as Table 4 .

Each data packet from the same node has a unique number. The source node id and data number represent a unique data packet in whole network. The head id represents the head node of forwarding this data packet. The next hop id1 and next hop id2 represent the next forwarder nodes which cost value is the two largest among the all neighbor head nodes at upper layer. The cost value of next hop id1 is larger than next hop id2. Residual energy is the current energy of head node of forwarding data. Forwarding ratio is the delivery ratio of forwarding head node.

If a cluster head receives no messages from other head nodes at upper layer, it will send the data to neighbor heads at same layer. The head nodes received data packet will compare its id to next hop id1 and next hop id2. If node's id is next hop id1 and the data packet is not recorded, it will forward the data packet immediately. Otherwise, if node's id is next hop id2 and data packet is not recorded, it will forward the data packet after waiting for a certain time. During the waiting time, the forwarder will drop the data packet if it receives the same data packet. The forwarding node records 10 data packet forwarded recently. If node's id is neither next hop id1 nor next hop id2, the data packet will be dropped. The procedure of next forwarder selection is shown as algorithm 2 .

3.4.3. Maintenance and Update Method of Clusters. The structure of underwater wireless sensor network is dynamic. The nodes move randomly with water current. Therefore, the structure of clusters may change at any time. A recovery mechanism is proposed in this paper.

For member nodes, when the member node fails to send the data to its cluster head, that means it has moved out of its cluster. In this case, it will monitor data packets from cluster heads. Once hearing a data packet of cluster head, it will join this cluster according to the information in data packet.

Each cluster head calculates the average energy of the its cluster. When its energy is lower than average energy, cluster is reformed in this layer and routing information is updated.

For the whole network, at each round of data collection, sink nodes expect the delivery ratio of next round. Once the delivery ratio is less than $70 \%$ of expected, the sink nodes will broadcast reformation message to the nodes. The underwater sensor nodes will rebuild the clusters.

\section{Simulations and Discussions}

In this section, the performance of EERBLC is evaluated and compared with DBR and EEDBR. The simulations are implemented using MATLAB 7.0. The same number of nodes are used in all simulations for fair comparison of EERBLC, DBR and EEDBR. 400 sensor nodes are deployed in a $500 \mathrm{~m}$ x $500 \mathrm{~m}$ x $500 \mathrm{~m}$ 3-D area. Initial energy of each sensor node is 5 joules. Power consumption of node in sending and receiving of data is $2 \mathrm{~W}$ and $0.1 \mathrm{~W}$, respectively. Transmission range of each sensor node is $100 \mathrm{~m}$. In the simulations of EERBLC, depth of each layer is defined at $80 \mathrm{~m}$, and 80 nodes are randomly deployed in each layer. All nodes 
TABLE 4: Format of data packet.

\begin{tabular}{llllllll}
\hline Type of packet & Data number & Source node id & Head id & Next hop id1 & Next hop id2 & Data & Residual energy Forwarding ratio \\
\hline
\end{tabular}

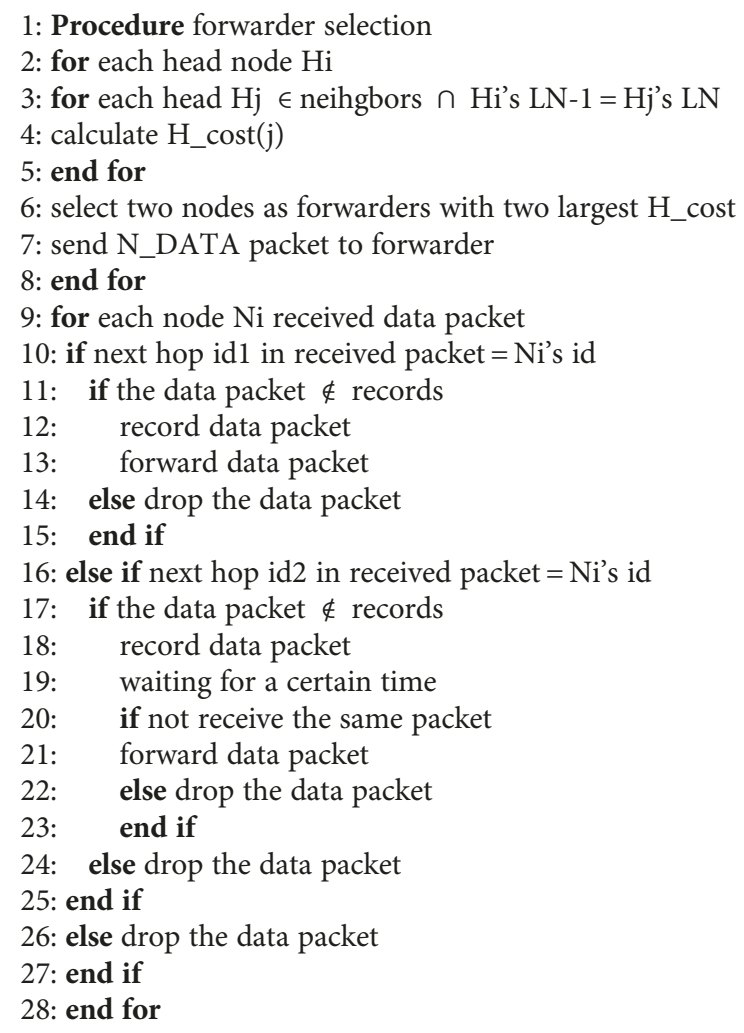

Algorithm 2: Next forwarder selection algorithm.

move with water currents from $2-3 \mathrm{~m} / \mathrm{sec}$ in the horizontal directions. Two buoys are deployed at the surface and used as sinks in order to collect the data packets from the sensor nodes. Every sensor node can hold 10 data packets in its buffer. Size of a data packet is 200 bytes, size of N_HELLO message is 5 bytes, size of N_COM message is 6 bytes, size of N_JOIN message is 5 bytes. Frequency of the carrier acoustic signal is $10 \mathrm{kHz}$. Simulation parameters are given in Table 5. The final simulation results are taken as an average of 5 different results.

In this work, the performance of protocols is evaluated in terms of stability period, network lifetime, throughput, delivery ratio, energy consumption and end-to-end delay. Stability period is defined as the duration till the first node dies in the whole network. For evaluating the performance of stability, the comparison simulations of DBR, EEDBR and EERBLC are implemented. All parameters of simulations are shown as Table 5 . The final simulation results are shown in Figure 3, which are taken as an average of 5 different results. In the DBR protocol, the first nodes died at 50s approximately. In the EEDBR and EERBLC protocol, the first nodes both died at $100 \mathrm{~s}$ approximately. In the DBR, a large number of redundant data packets are generated, and residual energy of node is not considered. That leads to premature death of nodes. In the EEDBR and EERBLC, energy is a major
TABle 5: Parameters of simulations.

\begin{tabular}{lc}
\hline Parameters & Values \\
\hline Monitoring area & $500 \mathrm{~m} \times 500 \mathrm{~m} \times 500 \mathrm{~m}$ \\
Number of nodes & 400 \\
Initial energy of nodes & 5 joule \\
Data packet size & 200 \\
Communication rang & $100 \mathrm{~m}$ \\
Number of sink nodes & 2 \\
$\alpha, \beta, \gamma, \theta, \mu, \varepsilon$ & $1,1,0.7,0.3,0.6,0.4$ \\
\hline
\end{tabular}

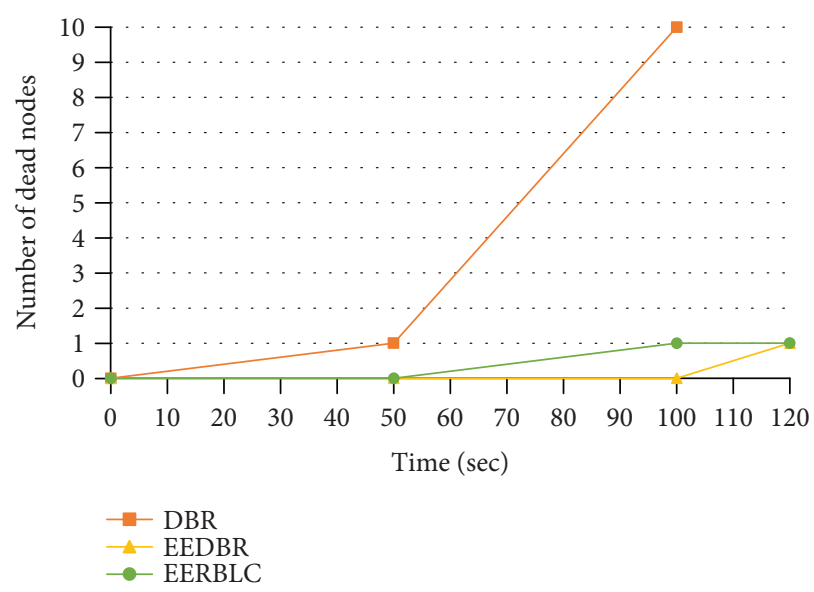

Figure 3: Comparison of stability period.

consideration factor during the forwarding data. Especially, EERBLC uses clustering technology to reduce the number of forwarding data. Hence, the time of first dead node is later than DBR.

Network lifetime is defined as the duration till the all nodes die in the whole network. Figure 4 demonstrates the network lifetime of three protocols in random topologies. The simulation results show that lifetime of DBR, EEDBR and EERBLC are about 1600s, 1800s and 2000s, respectively. Due to the redundant data and retransmitting, the lifetime of DBR is shortest in three protocols. In EEDBR, problems of energy and redundant data are improved to some extent, so its lifetime is longer than DBR. Clustering technology used in EERBLC saves the energy. And energy balance is taken into account during the routing. So, EERBLC has the longest lifetime among the three protocols.

Throughput is defined as the number of data packets successful received at sink nodes. Figure 5 shows the throughput simulation results of three protocols. The results show that DBR receives about 95,000 packets, EEDBR receives about 86,000 packets, and EERBLC receives about 70,000 packets during 2000s. DBR receives most packets due to large number of redundant data. EEDBR reduces the number of redundant data to some extent, so throughput of EEDBR is less than DBR. In EERBLC, because cluster heads aggregate data, 


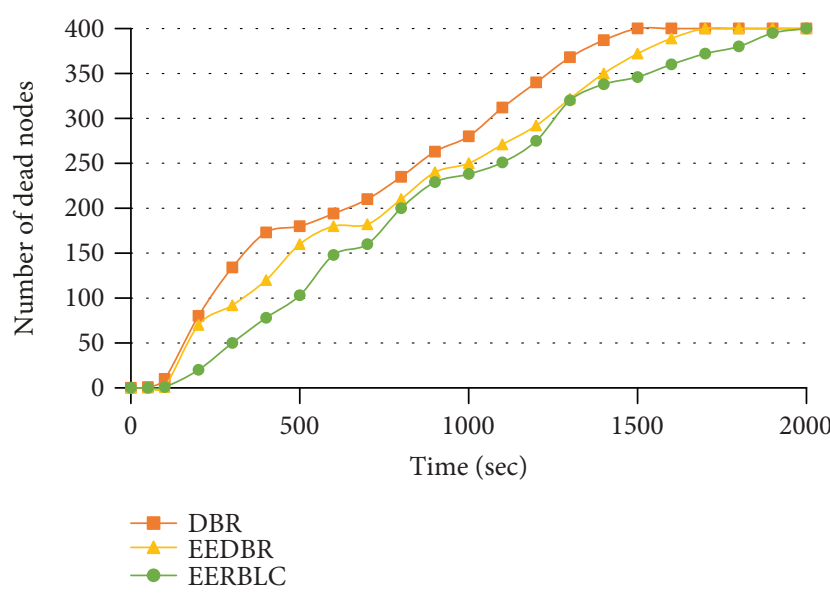

Figure 4: Comparison of network lifetime.

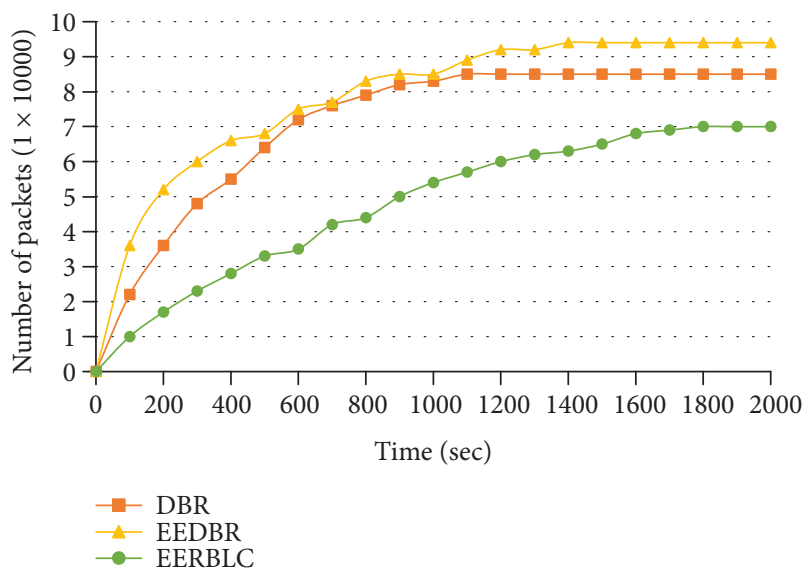

Figure 5: Comparison of throughput.

the number of data packets is reduced significantly. Hence the throughput of EBECRP is less than DBR and EEDBR.

Delivery ratio is defined as the ratio of the number of packets successfully received at the sink nodes to the number of packets transmitted from the source nodes. The comparison simulations are implemented to evaluate the performance of delivery ratio in DBR, EEDBR and EERBLC. The results are shown in Figure 6. Delivery ratio of DBR, EEDBR and EERBLC are $92 \%, 89 \%$ and $96 \%$, respectively. The delivery ratio of DBR is higher than EEDBR, because DBR makes packets transmitted redundantly where multiple paths are followed to reach the sink node. For avoiding redundant data, EEDBR reduces the forwarding nodes. That leads to decline of delivery ratio of EEDBR. The data are aggregated effectively in EERBLC, and forwarding ratio is considered during the selection of forwarding node. That leads to more data packets received by sink nodes in EERBLC. So, EERBLC has the highest delivery ratio among the three protocols.

Energy consumption is the indicator to the network performance, and it reflects the status of network lifetime implicitly. Lower consumption energy causes longer lifetime of network. Figure 7shows the simulation results of energy consumption. The energy consumption of DBR is higher than the other two protocols due to overmuch forwarding

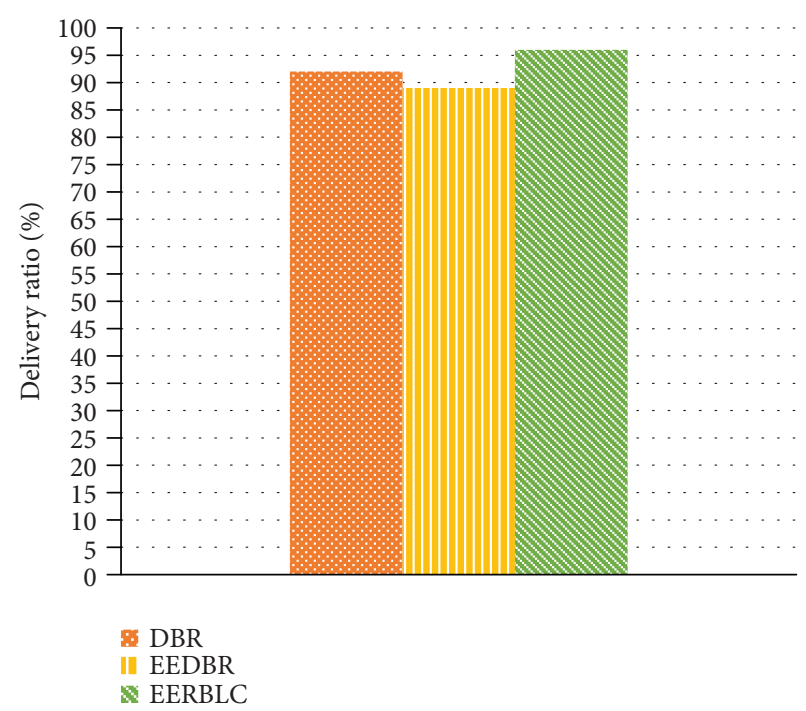

FIgURE 6: Comparison of delivery ratio.

nodes and redundant packets' transmissions. EEDBR selects forwarder based on the depth and the residual energy. It avoids the number of forwarding nodes. Moreover, in EEDBR, due to the priority assignment technique, repeat transmissions of the same data packets are reduced significantly. Hence, energy consumption of EEDBR is less than DBR. EERBLC uses the clustering technology to balance load of the whole network which results in balanced energy consumption and longer lifetime. In the network initial phase, clusters formation needs to consume more energy, therefore, the residual energy of EERBLC is less than EEDBR at first $300 \mathrm{~s}$. However, the energy consumption is less than other two protocols after initial phase.

End-to-end delay is defined as the average time which data packets are received by sink nodes from source nodes. DBR, EEDBR and EERBLC are compared by simulations. The results are shown in Figure 8. Because a certain holding time is needed before forwarding, DBR has the longest endto-end delay in the three protocols. Priority mechanism is adopted during the forwarding in EEDBR. The forwarder with highest priority transmits the packet immediately. Therefore, the delay is reduced. The data are aggregated effectively in EERBLC, so the reduction of data packets reduces the propagation time. Furthermore, link quality is considered during the selection of forwarding node, retransmissions are restricted effectively. So, EERBLC has the least end-to-end delay among the three protocols.

From the above simulation results, we can see that EERBLC has the longest network lifetime, highest delivery ratio and least end-to-end delay with the lowest energy consumption in the three protocols. It proves that EERBLC is an effective routing protocol for UWSNs.

\section{Conclusions}

It is hard to replace the batteries of underwater sensor nodes, therefor, energy efficiency is one of research hotspots in underwater wireless sensor networks. In this paper, a 


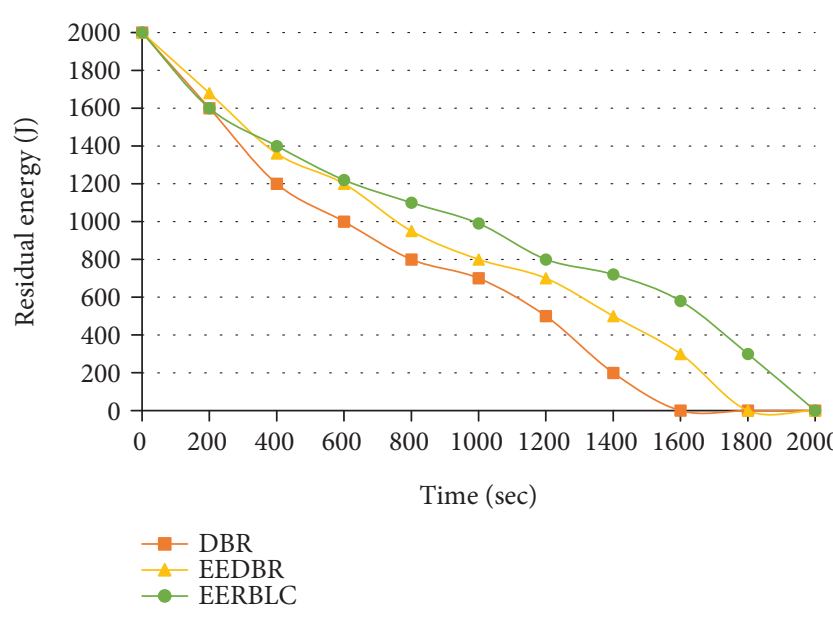

FIgURE 7: Comparison of energy consumption.

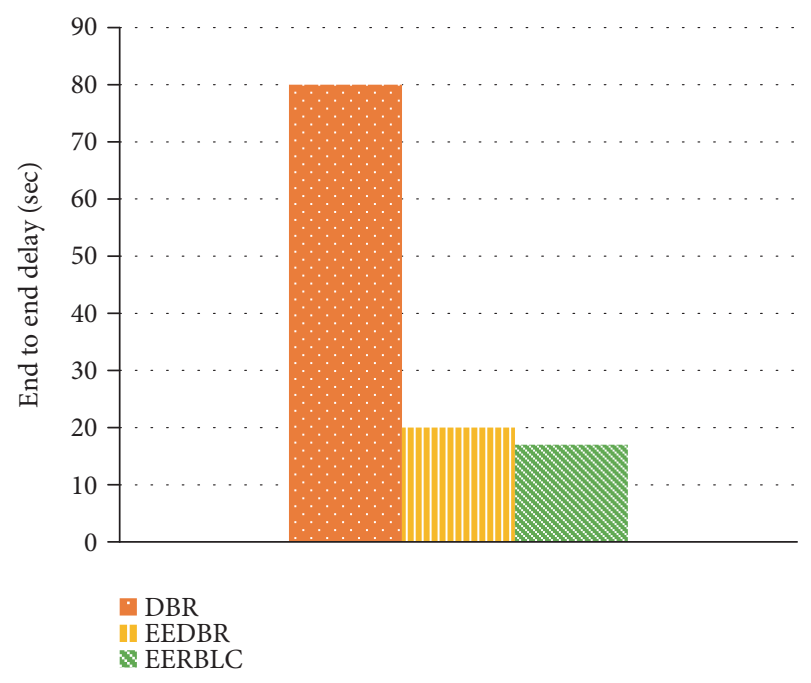

Figure 8: Comparison of end-to-end delay.

localization-free routing protocol, named energy efficient routing protocol based on layers and clusters (EERBLC) is proposed. This protocol aims to solve problems of high energy consumption, long end-to-end delay and high error rate. In this protocol, underwater monitoring area is layered, and then the sensor nodes are clustered at each layer. EERBLC includes three phases: cluster formation, transmission routing, maintenance and update of clusters. In the cluster formation phase, a new unequal cluster method suitable for UWSNs is proposed to solve "hotspot" problem. And, EERBLC improves the cluster head selection algorithm. A multi-objective optimization technique is introduced to calculate the cost value for each candidate head. In the transmission routing phase, EERBLC utilizes the forwarding ratio and the residual energy of sensor nodes as routing metrics. Finally, Intra and inter cluster updating method is presented. Through simulations, the EERBLC protocol is compared to DBR and EEDBR routing protocols in UWSNs. The results prove that EERBLC can effectively improve the performance in terms of network lifetime, energy consumption, delivery ratio, throughput and end-to-end delay.

\section{Data Availability}

The data used to support the findings of this study are available from the corresponding author upon request.

\section{Conflicts of Interest}

The authors declare that there is no conflict of interest regarding the publication of this paper.

\section{Acknowledgments}

This work is supported by the Fundamental Research Funds for the Central Universities under Grant No. N172304027.

\section{References}

[1] J. Heidemann, M. Stojanovic, and M. Zorzi, "Underwater sensor networks: applications, advances and challenges," Philosophical Transactions, vol. 370, no. 1958, pp. 158-175, 2012.

[2] G. Tuna and V. C. Gungor, "A survey on deployment techniques, localization algorithms, and research challenges for underwater acoustic sensor networks," International Journal of Communication Systems, vol. 30, no. 17, article e3350, 2017.

[3] E. Felemban, F. K. Shaikh, U. M. Qureshi, A. A. Sheikh, and S. B. Qaisar, "Underwater sensor network applications: a comprehensive survey," International Journal of Distributed Sensor Networks, vol. 11, no. 11, Article ID 896832, 2015.

[4] H. Mythrehee and A. Julian, "A cross layer UWSN architecture for marine environment monitoring," in 2015 Global Conference on Communication Technologies (GCCT), pp. 211-216, Thuckalay, India, April 2015.

[5] M. R. Ahmed, M. Aseeri, M. S. Kaiser, N. Z. Zenia, and Z. I. Chowdhury, "A novel algorithm for malicious attack detection in UWSN," in 2015 International Conference on Electrical Engineering and Information Communication Technology (ICEEICT), pp. 1-6, Dhaka, Bangladesh, May 2015.

[6] M. R. Ahmed, S. M. Tahsien, M. Aseeri, and M. Shamim Kaiser, "Malicious attack detection in underwater wireless sensor network," in 2015 IEEE International Conference on Telecommunications and Photonics (ICTP), pp. 1-5, Dhaka, Bangladesh, December 2016.

[7] S. Srinivas, P. Ranjitha, R. Ramya, and G. Kumar Narendra, "Investigation of oceanic environment using large-scale UWSN and UANETs," in 2012 8th International Conference on Wireless Communications, Networking and Mobile Computing, pp. 1-5, Shanghai, China, September 2013.

[8] C. C. Kao, Y. S. Lin, G. D. Wu, and C. J. Huang, "A comprehensive study on the internet of underwater things: applications, challenges, and channel models," Sensors, vol. 17, no. 7, p. 1477, 2017.

[9] M. Li, X. Du, K. Huang, S. Hou, and X. Liu, "A routing protocol based on received signal strength for underwater wireless sensor networks (UWSNs)," Information, vol. 8, no. 4, p. 153, 2017.

[10] P. Jiang, Y. Feng, F. Wu, S. Yu, and H. Xu, "Dynamic layered dual-cluster heads routing algorithm based on krill herd optimization in UWSNs," Sensors, vol. 16, no. 9, p. 1379, 2016.

[11] M. Jouhari, K. Ibrahimi, and M. Benattou, "New greedy forwarding strategy for UWSNs geographic routing protocols," in 2016 International Wireless Communications and Mobile Computing Conference (IWCMC), pp. 388-393, Paphos, Cyprus, September 2016. 
[12] Z. Zhou, B. Yao, R. Xing, L. Shu, and S. Bu, "E-CARP: an energy efficient routing protocol for UWSNs in the internet of underwater things," IEEE Sensors Journal, vol. 16, no. 11, pp. 4072-4082, 2016.

[13] M. Aslam, F. Wang, Z. Lv et al., "Energy efficient cubical layered path planning algorithm (EECPPA) for acoustic UWSNs," in 2017 IEEE Pacific Rim Conference on Communications, Computers and Signal Processing (PACRIM), pp. 1-6, Victoria, BC, Canada, August 2017.

[14] P. Xie, J. H. Cui, and L. Lao, "VBF: Vector-Based Forwarding Protocol for Underwater Sensor Networks," in NETWORKING 2006. Networking Technologies, Services, and Protocols; Performance of Computer and Communication Networks; Mobile and Wireless Communications Systems. NETWORKING 2006. Lecture Notes in Computer Science, vol 3976, F. Boavida, T. Plagemann, B. Stiller, C. Westphal, and E. Monteiro, Eds., pp. 1216-1221, Springer, Berlin, Heidelberg, 2006.

[15] N. Nicolaou, A. See, and P. Xie, "Improving the robustness of location-based routing for underwater sensor networks," in OCEANS 2007 - Europe, pp. 1-6, Aberdeen, UK, June 2007.

[16] S. M. Mazinani, H. Yousefi, and M. Mirzaie, "A vector-based routing protocol in underwater wireless sensor networks," Wireless Personal Communications, vol. 100, no. 4, pp. 15691583, 2018.

[17] M. R. Khosravi, H. Basri, and H. Rostami, "Efficient routing for dense UWSNs with high-speed mobile nodes using spherical divisions," Journal of Supercomputing, vol. 74, no. 2, pp. 696-716, 2018.

[18] J. M. Jornet, M. Stojanovic, and M. Zorzi, "Focused beam routing protocol for underwater acoustic networks," in Proceedings of the 3rd ACM International Workshop on Wireless Network Testbeds, Experimental Evaluation and Characterization (WuWNeT'08), pp. 75-82, San Francisco, CA, USA, September 2008.

[19] F. A. Salti, N. Alzeidi, and B. R. Arafeh, "EMGGR: an energyefficient multipath grid-based geographic routing protocol for underwater wireless sensor networks," Wireless Networks, vol. 23, no. 4, pp. 1301-1314, 2017.

[20] J. Jiang, G. Han, H. Guo, L. Shu, and J. J. P. C. Rodrigues, “Geographic multipath routing based on geospatial division in duty-cycled underwater wireless sensor networks," Journal of Network and Computer Applications, vol. 59, pp. 4-13, 2016.

[21] R. W. L. Coutinho, L. F. M. Vieira, and A. A. F. Loureiro, "DCR: depth-controlled routing protocol for underwater sensor networks," in 2013 IEEE Symposium on Computers and Communications (ISCC), pp. 453-458, Split, Croatia, July 2013.

[22] H. Yan, Z. Shi, and J. Cui, "DBR: depth-based routing for underwater sensor networks," in NETWORKING 2008 Ad Hoc and Sensor Networks, Wireless Networks, Next Generation Internet. NETWORKING 2008. Lecture Notes in Computer Science, vol 4982, A. Das, H. K. Pung, F. B. S. Lee, and L. W. C. Wong, Eds., pp. 16-1221, Springer, Berlin, Heidelberg, 2008.

[23] A. Wahid and D. Kim, "An energy efficient localization-free routing protocol for underwater wireless sensor networks," International Journal of Distributed Sensor Networks, vol. 8, no. 4, Article ID 307246, 2012.

[24] M. Ayaz and A. Abdullah, "Hop-by-hop dynamic addressing based (H2-DAB) routing protocol for underwater wireless sensor networks," in 2009 International Conference on Information and Multimedia Technology, pp. 436-441, Jeju Island, South Korea, December 2009.
[25] S. Basagni, C. Petrioli, R. Petroccia, and D. Spaccini, "CARP: a channel-aware routing protocol for underwater acoustic wireless networks," Ad Hoc Networks, vol. 34, pp. 92-104, 2015.

[26] Z. ying, S. Hongliang, and Y. Jiancheng, "Clustered routing protocol based on improved K-means algorithm for underwater wireless sensor networks," in 2015 IEEE International Conference on Cyber Technology in Automation, Control, and Intelligent Systems (CYBER), pp. 1304-1309, Shenyang, China, June 2015.

[27] A. Majid, I. Azam, A. Waheed et al., "An energy efficient and balanced energy consumption cluster based routing protocol for underwater wireless sensor networks," in 2016 IEEE 30th International Conference on Advanced Information Networking and Applications (AINA), pp. 324-333, Crans-Montana, Switzerland, March 2016.

[28] A. Khan, N. Javaid, I. Ali et al., "An energy efficient interference-aware routing protocol for underwater WSNs," KSII Transactions on Internet and Information Systems, vol. 11, no. 10, pp. 4844-4864, 2017.

[29] A. Khan, I. Ali, and H. Mahmood, "A localization-free variable transmit power routing protocol for underwater wireless sensor networks," in Advances in Network-Based Information Systems. NBiS 2017. Lecture Notes on Data Engineering and Communications Technologies, vol 7, L. Barolli, T. Enokido, and M. Takizawa, Eds., pp. 136-147, Springer, Cham, 2018.

[30] F. Hidoussi, H. Toral-Cruz, D. E. Boubiche et al., "PEAL: power efficient and adaptive latency hierarchical routing protocol for cluster-based WSN," Wireless Personal Communications, vol. 96, no. 4, pp. 4929-4945, 2017.

[31] M. Khanafer, I. Al-Anbagi, and H. T. Mouftah, "An optimized cluster-based WSN design for latency-critical applications," in 2017 13th International Wireless Communications and Mobile Computing Conference (IWCMC), pp. 969-973, Valencia, Spain, June 2017.

[32] S. Krishnamoorthy, "Enhanced adaptive clustering mechanism for effective cluster formation in WSN," International Journal of Current Engineering and Scientific Research, vol. 4, no. 9, p. 8, 2017.

[33] S. Mondal, P. Dutta, S. Ghosh et al., "Energy efficient rough fuzzy set based clustering and cluster head selection for WSN," in 2016 2nd International Conference on Next Generation Computing Technologies (NGCT), pp. 439-444, Dehradun, India, October 2017.

[34] G. Tuna, "Clustering-based energy-efficient routing approach for underwater wireless sensor networks," International Journal of Sensor Networks, vol. 27, no. 1, pp. 26-36, 2018.

[35] S. Rani, S. H. Ahmed, J. Malhotra, and R. Talwar, "Energy efficient chain based routing protocol for underwater wireless sensor networks," Journal of Network and Computer Applications, vol. 92, pp. 42-50, 2017.

[36] N. Goyal, M. Dave, and A. K. Verma, "Improved data aggregation for cluster based underwater wireless sensor networks," Proceedings of the National Academy of Sciences, India Section A: Physical Sciences, vol. 87, no. 2, pp. 235-245, 2017.

[37] D. Das and P. M. Ameer, "Energy efficient geographic clustered multi-hop routing for underwater sensor networks," in TENCON 2017 - 2017 IEEE Region 10 Conference, pp. 409414, Penang, Malaysia, November 2017.

[38] P. Jiang, J. Liu, and F. Wu, "Node non-uniform deployment based on clustering algorithm for underwater sensor networks," Sensors, vol. 15, no. 12, pp. 29997-30010, 2015. 


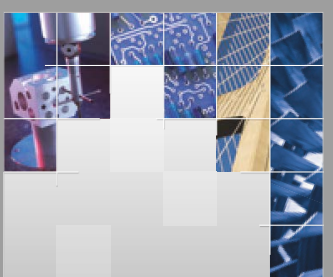

\section{Enfincering}
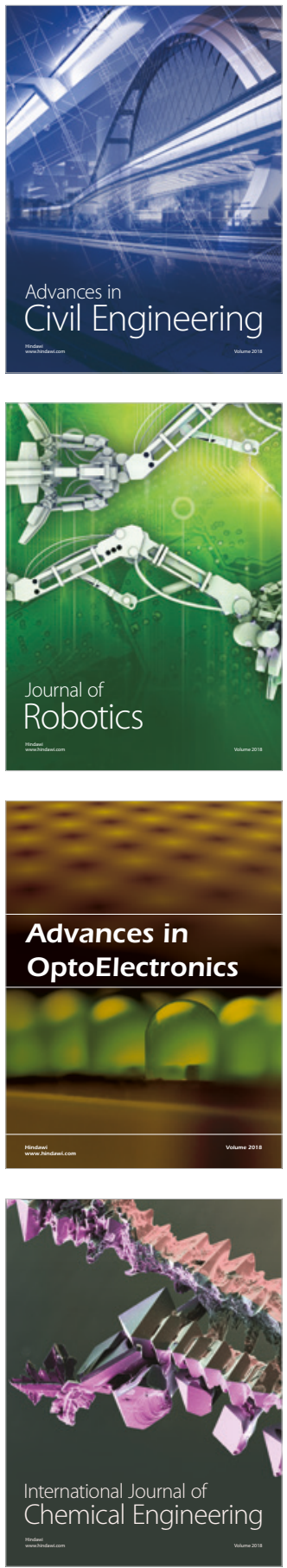

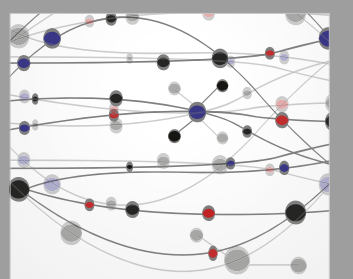

\section{Rotating \\ Machinery}

The Scientific World Journal

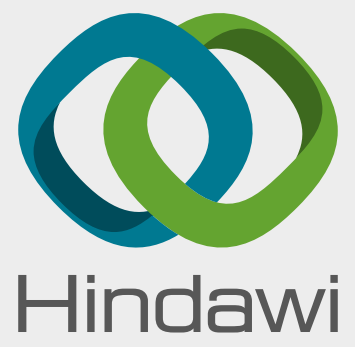

Submit your manuscripts at

www.hindawi.com
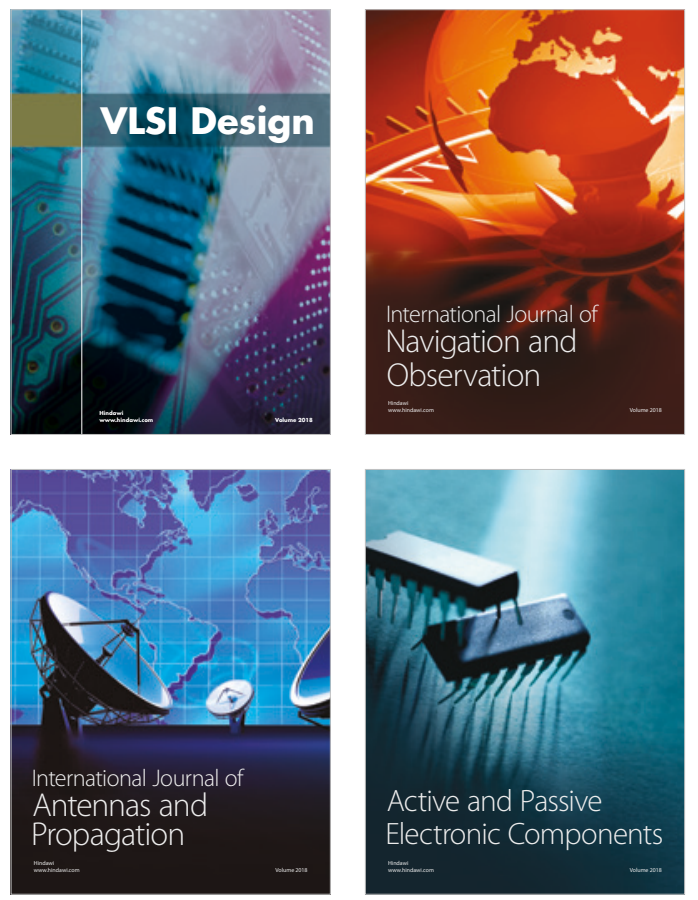
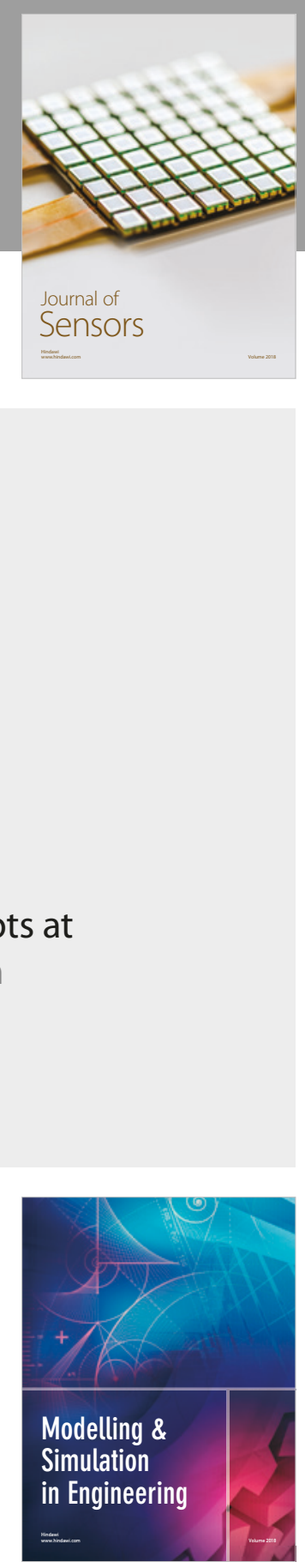

\section{Advances \\ Multimedia}
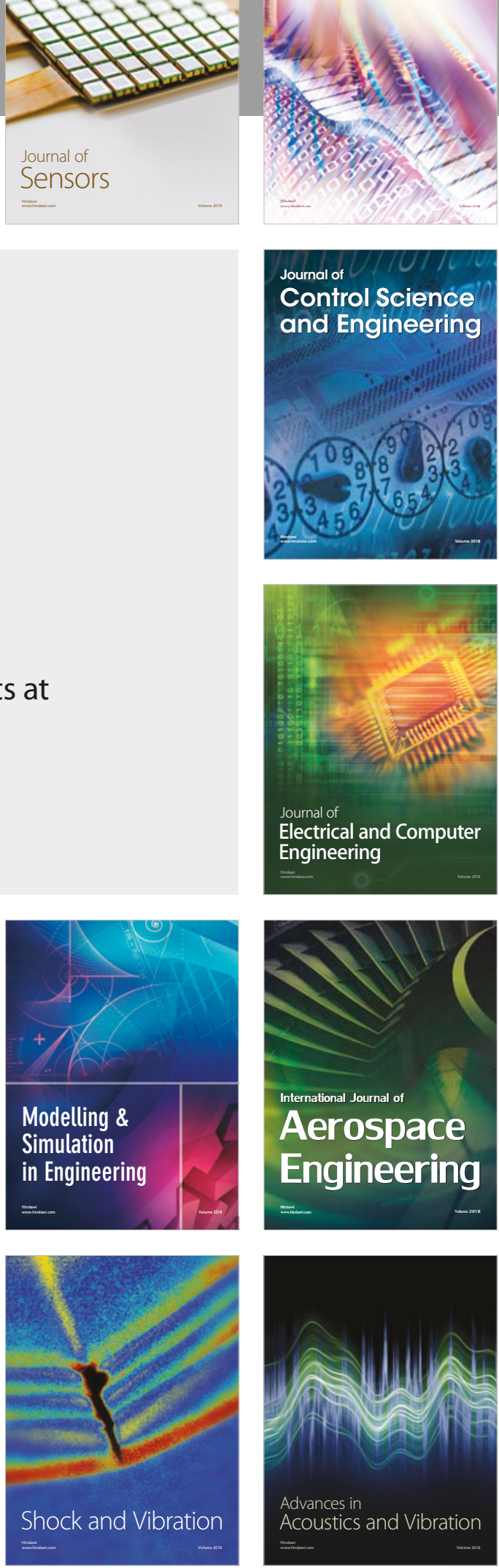